\title{
Comment on 'The bronze chime bells of the Marquis of Zeng: Babylonian biophysics in Ancient China' by E. G. McClain ${ }^{\dagger}$
}

\author{
Kenneth J. DeWoskin \\ University of Michigan
}

Study of the bells and other instruments uncovered in the fifth century BC tomb of the Marquis Yi of Zeng promises a contribution to the fields of history of science, technology, and culture of such magnitude that it should command the attention of experts in many fields around the world. Professor McClain makes this observation himself in an addendum to his article, circulated privately under the title 'Some personal observations of Chinese bells, with special attention to interiors'. 'Babylonian biophysics' is the first technical article on the bells by a non-Chinese writer that has garnered the interest of the Chinese. Huang Xiangpeng of the Central Academy of Music, a key researcher on the bells who is cited by McClain, plans to have the article translated into Chinese for distribution in China to musicologists. $\ddagger$

I began writing this article in Wuhan, the capital of Hubei province, site of the ancient state of Zeng. I have had the pleasure of making two study visits to the Hubei Provincial Museum, where the bells are now on display. The director of the museum, Professor Tan Weisi, is co-author of one of the key articles cited by McClain, analyzing the musical properties of the upper tier bells, especially McClain's Ib and Ic, the two sets on the west wall upper tiers. Writing on the subject of tuning systems with Tan is Feng Guangsheng, a young scholar of musicology with whom I have also had the pleasure of discussing the bells and their tunings.

Chen Zhongxing is director of the preservation department and is in charge of making replicas of the bells. Ordinarily, the availability of replicas would not be exciting, but there are two extraordinary things about these. First, the appearance of the replicas, even under close scrutiny, is impressive. They are visually indistinguishable from the authentic set. But the acoustical properties of the replicas are even more astonishing. The museum claims that both the lower guzheng (sui) and higher gupang ( $g u$ ) striking tones of the upper tier bells are within 5 cents of the original, and both tones of the lower bells are

\footnotetext{
† McClain (1985).

¥ Since my visit, a translation of the last section of the article, 'Part 3: Musicological significance', has appeared in a recently established journal of the prestigious Chinese Academy of Arts, Zhongguo yinyuexue (Musicology in China).
} 
within 10 cents. ${ }^{\dagger} \mathrm{Mr}$ Chen explained that the replicas were based on over 50,000 dimensional measurements, all integrated into computerized casting models. What I have seen of the replicas would validate McClain's assumption (1985, p. 153) that Chinese scholars have learned a great deal about the Zeng bell design. $\ddagger$ I cannot verify the absolute accuracy of the acoustical claims nor the detailed description of the replication process, but it is clear that great care went into the manufacture of the replicas. The second reason the replicas are of interest is that access to the original set is strictly limited. Below I will describe the impact of limited access on the state of scholarship, but even for the casual visitor to the museum, the original set is not easily examined. It is not badly displayed, but visitors are kept at a distance by a glass wall. The internal lighting is weak and the reflections are severe. Professor Tan has been kind enough to let me examine and play the replica set at length with striking bars and hammers which are themselves carefully executed replicas of originals found in the Marquis' tomb. Replicas of the bells have traveled all around China and have been used in attempted reconstructions of late Zhou dynasty musical performances.

Within a few years of the 1977 discovery of Marquis Yi's tomb and its musical treasures, the Chinese began to produce a steady stream of important publications of both a descriptive and interpretive nature. The final report on the dig and an adjacent second tomb $b^{\S}$ is nearing completion and will be published in about a year's time by the Hubei Provincial Museum. It is eagerly awaited because it will include, among many other important things, complete dimensional measurements for the bells, which have still not been published.

Japanese and western scholars have not had direct access to the original bell set or even to laboratory-quality recordings of the original set. They have had to confine their work to reinterpretations of the basic and incomplete data published by the Chinese. This is the result of an extremely protective policy the Chinese have maintained toward national treasures, especially archaeological treasures. What McClain lists as desiderata in furthering study of the bells in part exists, in part does not. There are, for example, high-quality recordings of each separate tone, but because of protective policies they have not been made available outside China. A full set of physical measurements have not yet been released on the primary bell set, although the measurements have been published for the smaller set in tomb 2. More complete physical measurements of the 65-bell set will be included in the final report mentioned above. Understanding this lack of access is fundamental to an evaluation of the corpus of non-Chinese scholarship on the Zeng bells. Little is known about the conditions under which the stroboscopic tuner analysis was done by Huang Xiangpeng (1981, pp. 22-53). Published data on the bell tunings are static data, providing no information on the changes of frequency over time and leaving significant questions about the psychoacoustical perception and hence musical aspects of the tones. At the same time, very interesting work of a technical nature

\footnotetext{
† The terms sui and gu describing the center lip strike point and the off-center lip strike point, respectively, were the terms originally preferred by Chinese publications on the bells and are the terms generally used by McClain. More recently, this nomenclature has been replaced by guzheng (literally 'lip center') and gupang (literally 'lip waist'). Note the typographical error gupgang for gupang in McClain (1985, p. 151). In passing, another confusing romanization error is bian-quing for bian-qing (twice on p. 49).

I It is interesting to note in this connection the attempts at casting a Chinese-style, two-pitch bell by Andre Lehr at the Royal Eijbouts Foundry. One of Dr Lehr's bells has been carefully analyzed by Thomas Rossing and his colleagues at Northern Illinois University and reported to the December 1986 meeting of the American Acoustical Society in Anaheim California.

$\S \mathrm{A}$ much smaller set of 33 bells was discovered in the second tomb, but preliminary indications are that these are purely decorative castings and cannot sound musical tones. The walls are very thick and, even though they are in excellent condition, do not sound. The stand had deteriorated completely.
} 
has been published by Chinese researchers in both Chinese and English, including the widely circulated study by Ma Chengyuan and others (1981) of the modal dynamics of the dual-pitched instruments based on laser holography. ${ }^{\dagger}$ Articles have been published on the metallurgical analysis of the bells, the casting techniques, the epigraphy, the aesthetics, and the musicology.

McClain is absolutely correct in identifying 'what has always seemed to be an excessive Chinese emphasis on the importance of music' (1985, p. 148). Virtually all major received texts, philosophical, historical, and poetic, from archaic China are replete with music, concerns about musical systems, music as an educating instrument, music as a component in ritual activity, music and pitches as keys to understanding of nature. Precisely this emphasis, however, is responsible for a high degree of mythologizing about music and musical instruments in early texts. One must be skeptical as to whether pitch-pipes were indeed used to establish measures of length (McClain, 1985; p. 169), whether pitch-pipes were used to measure volume of grain (p. 169), or whether bells evolved from grain scoops (p. 152).

These claims are enshrined in the canon of classical texts, but do they reflect historical facts? Confucius (551-479 BC) stressed the central role of music and ritual in maintaining social order. He lived at a time when the institutions and legitimacy of the Zhou dynasty were largely discredited, and he sought to establish a body of uniform practices which would restore the social and political order of the earlier years. The idea that a musical measure or a musical system stood at the center of a fair and reliable system of weights and measures, a well-wrought annual cycle of rituals, and proper code of moral and ethical action fit well into Confucian ideology as it evolved in the final centuries of the Zhou dynasty. Three centuries later, when Confucianism became the state religion of the court during the Han dynasty (206 BC-AD 220), rituals and music alleged to be authentic Zhou dynasty practices were reconstructed. The intent was to restore the order that flourished under the good governance of the Zhou. With these reconstructions, there developed elaborate schemes of correlations in which musical systems played a central structural role. The 12-pitch gamut was related to the 12 lunar cycles of the tropical year. The five intervals of the orthodox performance scale were related to the five phases. $\ddagger$ From these fundamental schemes of 12 and five, an elaborate edifice of relationships was developed to interpret natural phenomena, human behavior and political structure, and the processes of history.

There was a passionate commitment to 'regularities' and to 'wholeness'. Music was an ideal medium with which to demonstrate the importance of regularities and wholeness to cosmic order. The simple algorithm used to generate the chromatic scale demonstrated the whole-to-part relationship and showed how the one gave birth to the many. The reproducibility of pitches, the arithmetic beauty of the pitch calculations, and the demonstrable power of sympathetic resonance to make remote things interact were all of intense interest to Han thinkers. Music bore a heavy burden in Han proofs of natural principles. As a result, what Han recorders had to say about the music and the instruments of the preceding Zhou dynasty was strongly colored by the philosophy of their own times. A desire for wholeness in the correlation of pitch, time, and physical

\footnotetext{
'See also Jia Longsheng et al. (1981). A shorter English version of Ma's article was presented at the Metropolitan Museum of Art (June 2-3, 1980), and an English version was published in Chinese Music (1980).

I Five phases, commonly translated five elements, were central to all of the five-part correlations, including those in medicine, society, and material. For an expanded explanation of these correlations and a quotation from an early Han music bureau official, see DeWoskin (1982, pp. 67-69).
} 
metrological systems prompted Han records to grain scoops certified with pitch-pipes and standard lengths confirmed by pitch-pipes.

Similarly, many claims about the antiquity of instruments and systems must be scrutinized in the light of reliable evidence and not passed on uncritically. Even the existence of the Xia dynasty, for example, has not been archaeologically documented to the satisfaction of many scholars. ${ }^{\dagger}$ There is no solid evidential basis for specifying the Xia and Shang foot in centimeters to three decimal places (McClain, 1985; p. 170). McClain notes that the oldest pitch-pipe calculations are preserved in the writings of Sima Qian, the first century BC historian who compiled the Records of the Grant Historian (Shih-chi). The influence of Han thinking is evident in the mere desire of Sima Qian to reconstruct these measures. It is worth making explicit the point that he wrote $1000-2000$ years after the Shang and putative Xia dates. Not only do we not know, we cannot even imagine what evidence the Grand Historian might have had of their tuning systems and pipes. In fact, scholars today have a wealth of material evidence on the Shang compared to what Sima Qian had in his possession.

Han evidence cannot be used to prove much prior to the Han, which means that Sima Qian's claim for pitch-pipe measurements were Han figures, about 350 years after the Zeng bells were interred. Han numerologists had schemes that permitted them to project backwards through previous dynasties, and, depending on their numerically dictated regularities, determine many details of early ritual and musical practices. In other words, any variations from Han standards would have been projected variations, not documented variations, computed in relation to five phases (wuxing) numerology and sexagenary cycle dating (tiangan dizhi) ${ }^{\ddagger}$ The earliest archaeologically recovered qin zithers are the examples from the Zeng tombs. There is no material evidence of the qin from the fourteenth or fifteenth centuries BC. Oracle bone evidence is still based on a conjectural interpretation of the graph for 'music', and the oracle bones, in any case, do not predate the thirteenth century. There are no written records on oracle bones or any other media from the eighteenth century BC (McClain, 1985; p. 168). The epigraphic evidence cannot be used to prove the existence of hui string markers on the earliest qin and thus the antiquity of division by fifths and sixths (p. 168). Finally, the day count of the solar year at $3651 / 4$ does not necessarily date to 1000 years before the Marquis of Zeng (pp. 164-165). One bit of oracle bone evidence has been interpreted to be a day count between solstices, but it remains an uncertain interpretation. These sinological observations do not refute any of McClain's basic claims; they only seek to adjust his perspective on precedence and antiquity.

Discussions of Chinese tuning systems may be divided into those that deal with generation of the pitches and those that deal with musical structure or logic. Chinese discussions of tuning systems are often concerned with two orders, a generative order and a logical order. The gamut of 12 pitches has a generative order, the serial generations of the cycle of fifths process. The logical order follows ascending frequency, with alternating pitches marked yin and yang, and the annual calendric cycle overlayed on the full 12. A part of the logical order is the naming of sound, tonal relata and absolute pitches. The structure of the nomenclature is of special interest. McClain discusses many aspects of the Zeng bells, but he does not enter into the debate over generation. His original contributions are in the latter area of musical structure as documented by the

\footnotetext{
† For summary remarks by a proponent of the existence of the Xia, see Chang (1983).

$\ddagger$ For a discussion of the numerological systems and their use in musical, ritual, and historical argument, see DeWoskin (1982, pp. 43-84).
} 
bells, both the measured frequencies of the tones and the semantic pattern of the inscribed names.

Underlying his analysis of the tonal structure is the issue proposed by the subtitle; can the Zeng tonal system, attributed to the fourth century BC in China, be related to tonal systems and their attendant numerological apparatus from the ancient Middle East? In fact, even though there is a strong sense of national pride nurtured by the bells - Chinese scholars have trumpeted them as evidence of the early development of equal temperament in China-I have been asked by some young Chinese musicologists for readings on Babylonian music, apparently in response to McClain's search for an ancient 'Mesopotamian biophysics' in the foundation of the Zeng tonal structure.

The musicological problems focus on the tunings and are related to the special capacity of the bells to sound two musical tones. The Chinese called these parallel tones or dual tones. Because each bell sounds two notes, in performance their pitches are interleaved. The upper pitch of a given bell will be higher in frequency than the lower pitch of at least one smaller bell in the same gamut. That the dual tone capability of early Chinese bells was an intentional design was confirmed in an impressive way by the Zeng set. Each bell has two strike points marked, and they are marked with exact tone names. In addition to the strike point tone names, there are other tone names rendered in alternate pitch systems. Altogether, the tone and pitch names are inscribed with great superfluity, frustrating the search for a parsimonious logic or pattern to the Zeng musical system. This complexity is aggravated by the fact that the bell tones are interleaved. According to the analysis done at the museum, the inscriptions were all carved in the bronze bell bodies after the bells were cast. However close the cast bells were to their intended pitch, after casting they could be sounded and inscribed with a number of tone and pitch names. After carving, inscriptions were inlaid with gold, making the bells more precious and their inscriptions more legible.

Including the gift bo bell from the King of Chu, the set sounds 130 tones. But the inscribed tone names far outnumber the pitches. All of the bells have the two strike position tones marked on the recto side of the bell. The verso side of all of the bells of the lower and middle tier have additional inscriptions giving variant tone names in relation to other pitches. These reflect both regional differences in nomenclature and different points of reference to different pitch gamuts. On the top tier, as the bells are currently arrayed, only nine of the 19 bells have extended inscriptions on their verso side. In addition to inscriptions on the bells themselves, both the rack and the suspension hardware of the lower and middle tiers are frequently inscribed with tone names. These inscriptions on the stands and hanging fittings would presumably show where a particular bell was to be hung and with what fittings. Their existence suggests that the bells were somewhat portable and were intended to be dismantled and reassembled by people less familiar with them than the original designers and casters. On many bells, most of the flat surface is covered with inscriptions relating to the tone and pitch relations. The Zeng marquis and his musicians had a strong interest in the pitch systems. ${ }^{\dagger}$

Counting the inscribed tones on the lower and middle tiers, that is, counting the bells, racks, and fittings, there are over 700 names, several times the number of pitches that could be played. The upper tier set 1 has no verso inscriptions. Counting sets 2 and 3 , we have nine of the 15 bells with a single line, referring the pitch to a gong tonic on one of the 12 pitches. Interestingly, upper tier set 1 has balanced pairs of strike note

\footnotetext{
${ }^{\dagger}$ It has been argued that the top tier of belis was primarily an acoustics laboratory instrument and not used in performance (Huang, 1981; p. 23).
} 
inscriptions. That is to say, both strike points are referenced to the same basic $y \ddot{u}, z h i$, shang, or gong interval, with notational suffices attached to indicate the tonal displacement between the two notes. ${ }^{\dagger}$ This is suggestive of the logic with which the tonal system was organized. The following chart describes set 1 on the upper tier.

Set 1:

\begin{tabular}{lllllll} 
& \multicolumn{7}{c}{ Bell number } \\
& \multicolumn{1}{c}{2} & 3 & 4 & 5 & 6 \\
\cline { 2 - 6 } & $y u$ & $z h i Z$ & shangZ & zhi & yüZ & gong \\
Lower & $y \ddot{u} Z$ & $z h i J$ & shangJ & zhiZ & yüJ & gongZ
\end{tabular}

Even though the notational system is structurally a system of major thirds, only three of the six bells $(1,4$, and 6$)$ have that interval inclusive of the root. The other three have their suffixed inscriptions referenced to the root in different octaves.

Inscriptionally, the upper tier set 2 and set 3 are the exact opposite; on no bell does the root appear twice. But the notational suffixes are consistently used. Set 2 uses only shang and $y \ddot{u}$, in a three-bell pattern repeated for two octaves. The same pattern holds true for the top six bells of set 3 , except that they are referenced to gong and $z h$ i. For sets 2 and 3 we have the following arrangements.

Set 2:

\begin{tabular}{|c|c|c|c|c|c|c|}
\hline & & & $\mathrm{Be}$ & ber & & \\
\hline & 1 & 2 & 3 & 4 & 5 & 6 \\
\hline Tiqho- & $y \ddot{u} J$ & $y \ddot{u}$ & $y \ddot{u} Z$ & $y \ddot{u} J$ & $y \ddot{u}$ & $y \ddot{u} Z$ \\
\hline Lower & shang $Z$ & shangJ & shang & shang $Z$ & shangJ & shan \\
\hline
\end{tabular}

Set 3:

\section{Bell number}

\begin{tabular}{llllll}
2 & 3 & 4 & 5 & 6 & 7 \\
\hline
\end{tabular}

$\begin{array}{lllllll}\text { Higher } & \text { zhiJ } & \text { zhi } & \text { zhiZ } & \text { zhiJ } & \text { zhi } & \text { zhiZ } \\ \text { Lower } & \text { gongZ } & \text { gongJ } & \text { gong } & \text { gongZ } & \text { gongJ } & \text { gong }\end{array}$

the first bell of set 3 is anomalous, with shang and $y \ddot{u} Z$ marking the lower and higher pitches, respectively. Essentially the same pattern is evident in the paired strike pitches of every bell in the entire set, although the larger bell sets do not have the regularity of the upper three in this regard. The role of just thirds in the organization of the three small bell sets is undeniable. The argument here is that the structure of the nomenclature is homologous to the structure of the tuning system, and it suggests the centrality of just third in the generative and logical tuning system.

Examining the lower and middle tiers only, there are 45 bells, excluding the amusical bo dedicatory bell. Each bell on average has over 14 inscriptions, with a range from 9 to 17 . This count includes the basic two strike point names common to all bells and

\footnotetext{
$\dagger$ For purposes of reference, I will borrow linguistic convention and call the basic four intervals listed above 'roots'. I use $Z$ for the notational suffix zeng, indicating the pitch descends a major third below the root and $J$ for the notational suffix jue, indicating the pitch ascends a major third above the root.
} 
implicitly referenced to a single tonic. Beyond this primary context, on average a single tone fits into an additional 5 to 7 relational contexts. ${ }^{\dagger}$ It is this notational polysemy on the larger bells that has challenged scholars to find the internal logic of the Zeng bell tunings.

The advancement of casting technology between the Shang bells and the Zeng bells seems to have involved stretching the interval between the guzheng tone and the gupang tone. Shang bells show intervals of a major second or minor third, as McClain notes (1985, p. 154); in most Zeng bells the interval is a minor third (47) and in the remainder a major third. This interval need not, however, be a primary determinant of the overall tuning scheme, the relation of one bell to another. Put another way, the single bell guzheng to gupang interval does not completely constrain the logic of the overall tuning system. But, if in light of the inscriptional structure and McClain's revised analysis of the measured pitches, the case for a just tuning system based on major and minor third holds, we may argue that the concurrence of those key intervals in a single bell may have influenced the overall pitch systems. The technological advance from Shang intrabell intervals of a major second-minor third to that of Zeng bell intervals of a minor third-major third coincided with the introduction of a just third tuning strategy as an overlay on the traditional cycle of fifths strategy.

The suggestion that the Zeng bells served as an acoustical tonometer is of interest. The Chinese did, as McClain describes (1985, p. 163), have a great interest in studying vibration rates. It is not certain, however, that they ever sought exact counts of vibration rate, as McClain suggests in referring to Scheibler's beat count tonometer of the early nineteenth century. Chinese experiments in this area sought to correlate pitches to the configuration of cosmic $q i$ energy. Experiments like the one McClain describes using membrane-covered jars were meant to measure by sympathetic resonance. Correspondence of pitch, not absolute value, was the object of measurement. There is on the Zeng bell inscriptions a notable absence of numerical references. Throughout Chinese records, including highly arithmetic treatises on the pitches, the emphasis is on pitch ratios and correlations, not on absolute vibrational value. In other words, it was important to know that the Yellow Bell was indeed tuned to the absolute cosmic Yellow Bell pitch, but it was not important to know the absolute frequency of the Yellow Bell pitch.

This is a point of some importance in understanding early Chinese thinking and analyzing the logic of Chinese tuning systems. In general, the Chinese interest in numbers was an interest in the interrelatedness of things, not quantification and absolute numerical value. Needham, quoting the great sinologist Marcel Granet, notes that:

Numbers were manipulated as if they were symbols . . Numbers did not have the function of representing magnitudes; they served to adjust concrete dimensions to the proportions of the universe (Needham, 1956).

Hence, a pitch was not an abstract parameter but a correlate to cosmic absolutes. Tones were understood not as absolute values, but as relata within a variety of contexts, a pitch gamut, a seasonal ritual, a particular configuration of cosmic qi energy. The interest in relationship rather than absolute value is characteristic of the epistemology of regularity and order. Even the naming of a tone requires a context, so we find the multiplicity of tone name inscriptions on the Zeng bells.

\footnotetext{
${ }^{\dagger}$ These counts are based on the inscriptions as transcribed in 'Suixian Zeng Houyi mu zhongqing mingwen shiwen' ('Explanations of the inscriptional texts from the bell and chime sets of Zeng Marquis Yi's tomb in Suixian county') (Huang. 1981: pp. 3-13).
} 
McClain's discussion of features of the bell design that contributed to control of pitch and timbre brings out several important points bearing on the shear viability of such an instrument (1985, pp. 153-154). Features he notes are crucial for the bells to function as melodic instruments. One is the capacity of the Zhou style bells to sound a true fundamental rather than the subjective pitch or 'hum' tone below the strike note of a round carillon bell.

To the physical features McClain describes another can be added, the mei nipples which are cast on four plates. These have been discussed both in terms of their decorative and acoustical functions. On mature Zhou bells, there are almost always 36, arranged in four plates of nine. Within each plate is a three by three matrix, a scheme that probably had numerological significance in the ritual use of the bells. McClain suggests that the mei nipples are local loading devices, attenuating certain overtones and clarifying the separation of the two pitches (McClain, 1985; p. 153). Chinese scholars have argued elsewhere, on the basis of replica-casting experiments, that the mei nipples were dampers which hastened decay of the bell sound and permitted rapid sequential striking of notes without the need for mechanical damping or the confusion of unruly sustained ringing.

The Zeng bells make clear that distinct musical traditions existed in the several states of the late Zhou period. Tone names are expressed in systems of Zeng primarily, but also in systems of Zhou, Chu, Jin, Qi, and Shen (Huang, 1981; p. 23). ${ }^{\dagger}$ McClain notes the interesting disparity between complexity of tonal resources of the Zeng bells and the historical records. In historical records an orthodox ritual tradition of pentatonic music is preserved. There are occasional references to heptatonic relata. These references are critical, identifying such added and changed tones as corruptions of musical orthodoxy, and as corrupting of human nature. It is a surprise, then, to find such an abundance of tonal and modal perspectives in the Zeng bells, as McClain notes (1985, p. 160). They would surely have been regarded as very corrupt by the guardians of musical orthodoxy.

The received tradition places the most orthodox practices in the state of $\mathrm{Lu}$, in present-day Shandong Province, a great distance from Zeng and Chu. The Lu traditions would have been most precisely preserved in the Zhou tuning system. The house of Zhou was the royal house, making the regional music of $\mathrm{Lu}$ the state orthodoxy during the Zhou dynasty. That Lu musical and ritual traditions were regarded as the most orthodox is not unrelated to the fact that Confucius was born and schooled in Lu. Through the influence of historians in later centuries, Confucius became the transmitter of several such orthodoxies in the corpus of elite learning, including the use of ya 'elegant' language. It was three or four centuries after Confucius lived that the ritual standard were codified in the forms we have them reported in the dynastic histories. Among other things, these standards reflect a widespread penchant in ritual for simplicity and minimalization. In music, it meant fewer pitches were better than more pitches.

Confucius spoke of the music of Zheng, a state he often cited as an example of the wicked and depraved. Resorting to an intercultural parallel, he synasthetically linked the music of Zheng, with its added tones, to the color purple. Purple, a combination of colors, is in contrast to a primary color, either red or blue. The songs of Zheng confound the elegant ritual music. Purple detracts from red. The application of this principle to pitches, the combination versus the unitary, can be secn in the pitch names, binomes versus monomes, charted above (DeWoskin, 1982; pp. 92-94).

It is possible that the long-standing conflict between orthodox and deviant musical pitch traditions in Zhou China was not confined to a disagreement over pentatonic tonal

† The identification of Shen is conjectural. 
relata versus heptatonic, as has long been argued. ${ }^{\dagger}$ That is to say, there may have been more to the disagreement than the addition of two 'changed' notes generated by the orthodox algorithm. After all, the orthodox algorithm, called the San-fed sun-yi ("threepart subtraction-addition') permitted a theoretically infinite number of cycle of fifths generations within a single octave (DeWoskin, 1982; pp. 46-47). Indeed, the San-fen sun-yi was extended in practice beyond the pentatones to generate the orthodox 12-pitch gamut, and it was eventually extended during the Han by orthodox acousticians to as many as 60 pitches within an octave in various microtonal experiments. These extensions of the pentatones were not necessarily for musical ends, but they were in no way regarded as unorthodox by Han scholars.

What separated the orthodox and deviant may have been more fundamental, relating to the enrichment of the tone generation algorithmic to produce just thirds, directly from the tonic, second, fifth, and sixth, basic pentatones generated through cycles of fifths. By this means, just thirds would have been generated from an underlying four tones, discarding the third generated above the sixth and extending beyond the octave. The probable role of a just tuning system based on thirds has recently become a topic of discussion among Chinese musicologists. ${ }^{\ddagger}$ Now given McClain’s argument, this is a particularly inviting notion. The nomenclature as used in Han texts for these 'changed' notes mirrors the structure of the tone nomenclature on the Zeng bell inscriptions, although the characters are different. $\$$ The two unorthodox 'changed' intervals are named with prefixes on pitch names of the underlying pentatones. The only difference is that the third, in Han nomenclature, has been normalized with a single syllable name. The normalized Han name for the third, jue, is the same word used as a prefix to designate superior generation by a third in the Zeng inscriptions.

Some additional credence for the idea that a fundamentally different series of tonal relata existed is found in the Zuozhuan ('Chronicle of [historian] Zuo'), in which the qiyin, 'seven sounds', are listed as a distinct category for the wusheng, "five tones'. It is more likely that this division in nomenclature followed from a difference in generation process, not simply a difference in note count. ${ }^{*}$ In the years to come, there will be a careful reconsideration of all the received texts and the newly excavated inscriptional material in light of the broadened view of tuning logics that the Zeng bells have forced upon researchers.

Chinese scholars have stressed the contrasting regional nomenclatures inscribed on the Zeng bells. From the pitch names, it appears that the Zeng pitch nomenclature derived from the orthodox Zhou nomenclature, with some minor variations. But the bells also carry the complete gamut of Chu pitch names, which are completely distinct from the Zhou. The other states represented in the bells inscriptions have only one or two pitch names recorded. The major opposing forces at work in the formation of the Zeng musical systems are those of Zhou and Chu.

But it is important to remember that not only a regional but a social and ideological stratification correlated to these plural tuning traditions in early Chinese music. It is clear that the complexity of the Zeng tuning logic does not necessarily result from a

† For a careful review of this analysis of China's two ancient musical traditions, see Needham (1962, pp. 163-164).

I See, for example, Huang Xiangpeng's recently published article 'Zhongguo chuantong yindiao di shuliluoji guanxi wenti' ('The relation between mathematical logic and traditional Chinese mode-keys') (1986).

$\S$ Huang argues that the use of the term bian after the Han dynasty to describe a descending third interval was a Han error, since bian had the meaning of a descendant half-tone in the Zeng bell system.

I Zhao 23 and Zhao 25.

\# Needham \& Robinson remark that this division in nomenclature is 'strange' (Needham, 1962; p. 163). 
fundamentally complex understanding of a unified tuning system, nor does the inscriptional content of the bells derive from purely musical, aesthetic, or mathematical considerations. The complexity results, at least in part, from an environment of many musical traditions in which a small but culturally eclectic state sought to shape its own synthesis. The state of Zeng, politically so minor that its name was not even known before the discovery of the bells, was dominated musically and every other way by its powerful neighbor Chu. This is attested by the bo inscriptional bell, a gift from the Chu king to his vassal, the Marquis of Zeng. But Zeng asserted its independence from a culturally dominant neighbor by integrating competing systems into a more comprehensive system. This latter explanation relieves us of the need to resolve what appear to be contradictions and superfluities in both the Zeng bell inscriptions and the measured pitches. We can identify a primary algorithm that was functional in Zeng music and recognize much of the complexity as overlays of other acoustical and logical approaches, perhaps some based on fundamentally different processes of tone generation.

It is very difficult to confirm McClain's suggestion that there was cultural contact between Babylonia and China, and that Babylonian tuning logic influenced China's. The great antiguity of $\mathrm{Ur}(2800 \mathrm{BC})$ bears witness to the early origins of tunable string instruments in Mesopotamia, but there is no such comparable material evidence of transmission to China. Even as McClain introduces this suggestion, he describes a 'human biological bias' sustaining universals from culture to culture, period to period. How does one assess the common origin versus independent development question in McClain's example? Spiral of fifths tuning is found in both Babylonia and China. The biological bias, whether related to the biophysics of hearing or not, makes the question moot. With or without the influence of an earlier tradition, the same outcome would have occurred, because it is in part constrained by biological factors and in part by astronomical factors. The former, the bioacoustical concerns, are beyond my area of competence. But the latter are easily demonstrated.

For all earthly civilizations, the approximate equivalence of 12 lunar cycles to one solar cycle defines the 12 months of the tropical year. This simple astronomical fact refutes any case for cultural diffusion based on the recognition of the number 12 in calendrics. Beyond this there is the infinite richness of astronomical regularities visible to the entire world. The astronomies of diverse civilizations are likely to bear strong semblances to one another, but what about their astrologies? For well over a century, the likelihood of cultural transmission from the ancient Middle East to China has been argued on the basis of similarities in calendric and astrological systems. These arguments are based on a presumption that one can delineate a conceptual level of knowledge from a perceptual level, or a constitutive from a cognitive. That is, they assert that we can separate what our archaic ancestors conceived, or, perhaps conceptualized, from what they perceived in their apprehension of the heavens. Similar perceptions could have been independently derived, but what of shared conceptions? That becomes the argument for cultural diffusion, as opposed to independent development. The burden of these arguments is wholly the burden of delineating where the conceptual divides from the perceptual, what is constitutive and what is cognitive.

Alexander Marschak's interpretations of Upper Paleolithic inscriptions arc very provocative in the context of this distinction (1972). Marschak studies a range of inscriptions that show a degree of patterned regularity. Mostly notched or pitted bones, archeologists had generally relegated them non-significant decorative inscription. Marschak argues that these inscriptions have a certain sort of meaning, one that is 'time-factored, relational, and concerns process (Marschak, 1972; p. 119). He plots 
several inscriptions against the phases of the moon, arguing that the inscriptions were related to the recognition of periodicity of lunar cycles. The time-factoring geometry of these external phenomena shaped visual-kinesthetic, non-verbal cognitive aspects in hominid communication (Marschak, 1972; p. 118). Having forged this link between cognition and the constitution of a symbolic system, Marschak argues that gross astronomical phenomena were largely invariant for two million years over the entire earth, providing thereby a source of independently derived but structurally similar time-factoring cultures around the earth. The inscriptions Marschak studies and relates to lunar periodicity come from widely diverse locations and different periods.

Finally, Marschak notes that for man at primitive stages, the "primary observational dramas in the heavens are the lunar phases, their general direction of origin and disappearance, and the general westward motion of the moon in the sky'. More subtle patterns and cyclic activities of longer period require record keeping and mathematics before cultures could achieve mastery and were inclined to pay them attention (Marschak, 1972; p. 127).

How might a construction as abstract as a 12-pitch gamut cleave to a perception of external astronomical phenomena? In China, the 12 pitches are closely associated with the 12 lunations that made up the normal (i.e. non-intercalary) tropical year. The two are closely correlated in number schemes throughout the Imperial period. The 12 bell names according to the traditions of Zhou are first mentioned in the Yuehling ("monthly ordinances') and the Kuoyü ('tales of the states'). Neither text can reliably be dated prior to the end of the Zhou, but the key musical passage in the Kuoyü presents itself as a record of the sixth century $\mathrm{BC}$, and both texts clearly contain material of at least mid-Zhou provenance. The Zeng pitch names on the Zeng bells derive from a system kindred to the one articulated in the Kuoyü and Yuehling, a system that has been identified with the Zhou tradition (Huang, 1981; p. 23). With a full record of the contrasting Chu pitch names, the bells document that at least two competing systems existed prior to the fifth century BC, but each was a system of 12 .

Early mythic accounts tell of the discovery of the pitches in nature by Ling Lun, a minister of the Yellow Emperor. Ling Lun, whose name bespeaks structure and order, was sent westward according to the following account:

In ancient times, the Yellow Emperor ordered Ling Lun to make pitch standards. Ling Lun traveled from west of Daxia all the way to the northern slopes of the Ruanyü Mountains. He gathered bamboo from the valleys of Xieqi, and, selecting those pieces with chambers and walls of uniform thickness, he cut a section from between two nodes. The length of a section was 3.9 inches and he blew it. That sound he took to be the gong tone at the Yellow Bell pitch level. Blowing it, he said, 'That is good!' and proceeded to make the shortened sections in a series, creating twelve pitch-pipes.

He carried these back to the foot of Ruanyü Mountain where he listened to the songs of the male and female phoenix, in order to divide the twelve pitches. The male (yang) calls numbered six and the female $(y i n)$ called numbered six. Proper concord was achieved by their relation to the gong tone at the Yellow Bell pitch. The gong tone at the Yellow Bell pitch could generate all of the rest. Therefore, Ling Lung said that the gong tone at the Yellow-Bell pitch was the origin of all the yang and $y$ in pitches.

The Yellow Emperor subsequently ordered Ling Lun to join with Rong Jiang to cast twelve bronze bells. These would provide harmonious placements of the five tones and allow the harmonious symphony to be performed. In the middle of spring, on the twelfth day of the cycle of sixty, when the sum was in the lunar lodging Straddler, it was performed for the first time. He ordered it to be called Xianshi. ${ }^{\dagger}$

\footnotetext{
† Translated from the Spring and Autumn Annals of Mr. Lü: 5.8a-9a (239 BC). For further discussion, see DeWoskin (1982, pp. 59-62).
} 
The imprint of astronomical phenomena on this myth is unmistakable. The dual terms yang and yin are derived from the periodicity of the solar and lunar cycles. The Ling Lun story is consistent with other accounts about the discovery of the trigrams and hexagrams and discovery of the writing system. In the Chinese mind, this particular construction of an ordered symbolic set was derived directly from observation of nature. In other words, to the archaic Chinese mind, musical systems were primarily cognitive rather than constitutive, a system that was referential to observed periodicity in nature.

The Yuehling, as preserved in the early Han Book of Rites (Liji), lists each pitch as an attribute of one of 12 lunation-defincd seasons. Each festival marks off a fraction of the tropical year according to a particular yueh moon. Under each moon, the text presents a set of correlates, including horoscopic recommendations and proscriptions for human action as well as co-ordinates of the sun and movements of other celestial lights. Also included is one pitch from the gamut of 12 , felt to belong to that lunation. In this structure, the independent event is the lunation; the dependent one the pitch. If, from the chronology of this myth, we can infer the chronology of historical process, we can advance our argument that the 12-pitch gamut derived its structure from the calendar. In other words, time-factoring cognition of lunar periodicity, in fact, underlaid the construction of the gamut of pitches or at least its specific articulation in early Chinese texts.

The association between pitch and calendar was a durable one. Subsequent dynastic histories, written for over 2000 years, generally included a 'Treatise on the pitch-pipes and calendar'. Harmonic considerations correlated to calendric and numerological ones. The association between a pitch and a time supported an elaborate and formal use of music in seasonal rites. This use in turn programmed each pitch with ritual and seasonal associations. Neither the importance of 12 in the pitch structure nor the fact that pitches correlated to months is inconsistent with patterns of regularity in nature that presented themselves to the Chinese or to what we know of Chinese thinking at the earliest stages. It then becomes a problem to determine if there were necessarily any acoustical or biophysical factors in the emergence of the Chinese pitch gamut as a duodecimal structure. All astronomical and biological factors in the construction of the 12-pitch gamut argue for independent development of the Chinese musical and acoustical systems. A case for cultural diffusion must rely on cultural particulars in the music system beyond all of these avenues to musical knowledge.

One cannot argue that the prevalence of 60 and 360 in later Chinese acoustical thinking is truly irrelevant to the official Confusian cycle of fifths (McClain, 1985; p. 168). The cycle of 60 is the most fundamental time-counting cycle known in China. Its roots reach directly back to the earliest written records, the Shang oracle bones, where ritual practices followed a 10-day xun cycle. The 10-day cycle, eventually codified in the tiangan heavenly stems, was rotated with the 12-count dizhi earthly branches to create a sexagenary cycle for counting days, months, and years. At the same time, a 12-pitch gamut based on cycles of fifth tuning was established very early as orthodoxy, as was a five-tone performance scale. The fullest elaboration of a five-tone movable scale on a 12-step, fixed-pitch gamut produced 60 mode keys in which music could be performed. These 60 mode keys can be generated without reference to any tuning system outside the orthodox cycle of fifths. Numerologically, there was reinforcement for the musical expansion of the mode-key inventory. Each pitch was associated with an earthly branch, the cyclic processes of which expanded to a cycle of 60 as described above. In addition to the long history of sexagenary cycle counting, the factorability of 60 by all numbers from 1 to 6 and the factorability of 360 by 1 to 6 and $8,9,10$, and 12 made these numbers 
of probable theoretical interest to any culture that explored arithmetic expressions or tools for pipe- or string-dividing acoustic experiments.

The only remaining evidence for some form of cultural diffusion is the appearance of just thirds in the Zeng tunings. This is admittedly a departure from the cycle of fifths tunings specified in the ritual texts. It is an important feature of the Zeng bells, but in and of itself it is not conclusive evidence of a Pan-Eurasian tonal cosmology.

McClain's great erudition and experience in the reconstruction of ancient musical traditions has been very illuminating for scholars of early Chinese music. His propositions in this article will open new issues for debate over the next several years. His efforts to push the significance of the stroboscopic statistics beyond what their precision merits will have the salutary result of driving scholars to make more and more accurate measurements of the bells. What can be learned by acoustical measurements will ultimately be constrained by aging losses in the bells themselves, not by technical limitations of measuring instruments. But this remarkable set of bells still has many secrets to yield to modern researchers and will continue to attract a widening circle of attention.

\section{References}

Chang, K. C. (1983). In (D. K. Knightley, Ed.): The Origins of Chinese Civilization. Berkeley: University of California Press, pp. 503-507.

DeWoskin, K. J. (1982). A Song for One or Two: Music and the Concept of Art in Early China. Ann Arbor.

Huang, X. (1981). Yinyue Yanjiu (Musicological Research) 1.

Huang, X. (1986). Zhongguo yinyuexue (Musicology in China) 3, 9-27.

$\mathrm{Jia}$, L. et al. (1981). Jiang Han kaogu (Archaeology of the Jiang Han Area) 1, 19-23, 25-30.

Ma, C. (1980). Chinese Music 3.

Ma, C. (1981). Kaogu xuebao (Journal of Archaeology) 1.

Marschak, A. (1972). The Roots of Civilization: The Cognitive Beginnings of Man's First Art, Symbol, and Notation. New York: McGraw-Hill.

McClain, E. G. (1985). J. soc. biol. Struct. 8, 147-173.

McClain, E. G. (1986). Zhongguo yinyuexue (Musicology in China) 3, 96-112.

Needham, J. (1956). Science and Civilisation in China, Vol. 2. Cambridge: Cambridge University Press, pp. 287-288.

Needham, J. (1962). Science and Civilisation in China, Vol. 6.1. Cambridge: Cambridge University Press. 